\title{
Effect of three Intravitreal Injections of Aflibercept on the CNV Guided by Optical Coherence Tomography Angiography in Wet Age Related Macular Degeneration
}

\author{
Ahmad El-Sayed Hudieb \\ Ophthalmology department, Faculty of Medicine, Al-Azhar University, Cairo, Egypt \\ Corresponding author: Ahmed El-Sayed Hudieb, Email: ahmadhodieb2013@ gmail.com, Phone Number: 002-01015488588
}

\begin{abstract}
:
Background: age-related Macular Degeneration (AMD) is a progressive, degenerative disease that affects the macula and it isone of the most common leading causes of irreversible vision loss in old patients with age more thanfifty. Optical coherence tomography angiography (OCTA) can detect the presence of choroidal neovascularization (CNV), structural changes in AMD; moreover, changes in choroidal blood vessel flow.

Aim: to assess and compare structural retinal changes in patients with neovascular age-related macular degeneration (AMD) treated with $2 \mathrm{mg} / 0.1 \mathrm{ml}$ intravitreal aflibercept three times with an interval of one month each.

Patients and methods: twenty eyes of non-diabetic non-hypertensive ARMD patients were included. They received $2 \mathrm{mg} / 0.1 \mathrm{ml}$ intravitreal aflibercept three times with an interval of one month each. OCTA was done before the first injection and was repeated one month after the third injection.

Results: the area size and the GLD of the CNV had highly statistically significantly improved after $2 \mathrm{mg} / 0.1 \mathrm{ml}$ three intravitreal aflibercept injections.

Conclusion: aflibercept is an effective treatment in AMD; it can decrease the area size, GLD and thickness of the CNV. OCTA is an effective tool that can provide a noninvasive method in the diagnosis and follow up of the activity and anatomical changes in AMD.
\end{abstract}

Keywords: optical Coherence Tomography Angiography; Aflibercept; Wet Age Related Macular Degeneration; CNV; Macular thickness.

\section{Introduction}

Age-related Macular Degeneration (AMD) is the leading cause of irreversible vision loss among individuals aged 50 or older in developed countries. It can be classified into two types: dry (atrophic) AMD and wet (exudative) AMD ${ }^{(\mathbf{1})}$.

Advanced wet AMD is characterized by choroidal neovascularization $(\mathrm{CNV})$, the leakage ofnew blood vessels can cause edema and disorders in retinal architecture eventually causing blurred and distorted vision. If left untreated, the area of neovascularization can expand rapidly leading to the development of fibrous scarring. Recent studies show that within 3 years of onset of neovascularization, about $75 \%$ of untreated patients will present a visualacuity of $20 / 200$ or worse $^{(2)}$.

Several risk factors for AMD have beenidentified, such as advanced age, geneticpredisposition, and history of cataract surgery, smoking history and diabetes ${ }^{(3)}$.

Vascular Endothelial growth factor-A (VEGF-A) has been implicated to play a major role in the pathogenesis of the wet AMD ${ }^{(4)}$.

VEGF-A and placental growth factor (PGF) are members of the VEGF family of angiogenic factors which may act as potent mitotic factors, vascular permeability and chemotactic for endothelial cells ${ }^{(5)}$.
Since VEGF plays an important role in the pathogenesis of the choroidal neovascular membrane (CNVM), the treatment for exudative AMD has been revolutionized with intravitreal anti-VEGF therapy: Ranibizumab, Bevacizumab and Aflibercept ${ }^{(5)}$.

Aflibercept is a fusion protein with bindingdomains from native VEGF receptors, binds VEGF-A,VEGF-B, and placental growth factors 1 and 2 withhigh affinity. Ophthalmologic studies demonstrated that aflibercept suppresses choroidal neovascularization in patients with exudative age related macular degeneration with excellent visual outcomes ${ }^{(6)}$.

The introduction of intravitreal antivascular endothelial Growth factor (VEGF) blockade for neovascular age-related macular degeneration (AMD) in the last decade has had a significant beneficialimpact on patients with neovascular AMD $^{(7)}$.

Published studies hint at the optical coherence tomography angiography (OCTA) potential efficacy in the evaluation of common ophthalmologic diseases such age related macular degeneration (AMD), diabetic retinopathy, artery and vein occlusions, and glaucoma ${ }^{(8)}$. 
OCTA can detect changes in choroidal blood vessel flow and can elucidate the presence of choroidal neovascularization $(\mathrm{CNV})$ in a variety of conditions but especially in AMD. It provides a highlydetailed view of the retinal vasculature, which allows for accurate delineation of the foveal avascular zone (FAZ) ${ }^{(\mathbf{8})}$.

Further studies are needed to more definitively determine OCTA's utility in the clinical setting and to establish if this technology may offer a non-invasive option of visualizing the retinal vasculature in detail ${ }^{(\mathbf{8})}$.

\section{Aim of the study:}

The aim of this study is to assess and compare structural retinal changes in patients with neovascular age-related macular degeneration (AMD) treated with $2 \mathrm{mg} / 0.1 \mathrm{ml}$ intravitreal aflibercept three times with an interval of one month each.

\section{Patients and Methods}

This study is a prospective, non-invasive and non-randomized study. It included 20 eyes of non-diabetic non-hypertensive ARMD patients.

The study methods and goals were explained to each patient, and an informed consent was signed by all patients.

All patients were received a dose of $2 \mathrm{mg} / 0.1 \mathrm{ml}$ intravitreal aflibercept three times with aninterval of one month each.

OCTA was done before the first intravitreal aflibercept injection and it was repeated again one month after the third injection.

\section{Inclusion criteria:}

Neovascular age-related macular degeneration (AMD) patients.

\section{Results}

Table (1): Pre-Treatment greatest linear dimension, area and retinal thickness on top of CNVdescriptive of the study group.

\begin{tabular}{|l|c|}
\hline & Total $(\mathbf{N}=\mathbf{2 0})$ \\
\hline Pre-treatment greatest linear dimension $(\mathbf{G L D})(\mathbf{m m})$ & $2.69-7.29[4.36 \pm 1.84]$ \\
Choriocapillaries & $1.85-4.72[2.89 \pm 1.16]$ \\
Outer retina & \\
\hline Pre-treatment Area $(\mathbf{m m})$ & $0.56-3.27[1.41 \pm 1.13]$ \\
Choriocapillaries & $0.27-1.41[0.63 \pm 0.47]$ \\
Outer retina & $339.0-782.0[488.3 \pm 176.99]$ \\
\hline Pre-treatment retinal thickness on top of CNV $(\mathbf{u m})$ & \\
\hline
\end{tabular}

This table shows that the mean choriocapillaries $4.36 \pm 1.84$ and outer retinal $2.89 \pm 1.16$ of pretreatment greatest linear dimension (GLD), also choriocapillaries $1.41 \pm 1.13$ and outer retina $0.63 \pm 0.47$ of pre-treatment area, while pre-treatment retinal thickness on top of CNV488.3 \pm 176.99 .

Table (2): Post-treatment greatest linear dimension descriptive of the study group.
Exclusion criteria include:

1- Diabetic patients.

2- Hypertensive patients.

3- Advanced ocular co-morbidity with poorvision.

4- Previous intravitreal injections.

Pre-operative evaluation: \&diabetes mellitus).

2) Best corrected visual acuity.

3) Full ophthalmic examination.

4) Optical coherence tomography angiography (OCTA).

Operative:

All patients were received a dose of $2 \mathrm{mg} / 0.1 \mathrm{ml}$ intravitreal aflibercept three times with aninterval of one month each.

Post-operative \& follow up:

1- First day post-operative full ophthalmic examination after each intravitreal injection with recording of any complications after each intravitreal injection.

2- OCTA one month after the third intravitreal injection.

The study was approved by the Ethics Board of Al-Azhar University. Statistical analysis:

Statistical presentation and analysis of the present study was conducted using the mean, standard deviation, unpaired student t-test, paired $\mathrm{t}$-test, and chi0square tests by SPSS V17.
1) Systemic assessment (blood pressure 
Effect of three Intravitreal Injections of Aflibercept on the CNV Guided by Optical Coherence...

\begin{tabular}{|l|c|}
\hline Post-treatment greatest linear dimension $(\mathbf{G L D})(\mathbf{m m})$ & $1.40-4.92[2.99 \pm 1.35]$ \\
Choriocapillaries & $0.00-3.02[1.74 \pm 1.18]$ \\
Outer retina & \\
\hline Pre-treatment Area $(\mathbf{m m} 2)$ & $0.26-2.35[0.99 \pm 0.84]$ \\
Choriocapillaries & $0.00-1.02[0.40 \pm 0.39]$ \\
Outer retina & $224.0-355.0[279.0 \pm 56.90]$ \\
\hline Post-treatment retinal thickness on top of CNV (um) & \\
\hline
\end{tabular}

This table shows that the mean choriocapillaries $2.99 \pm 1.35$ and outer retinal $1.74 \pm 1.18$ of Post treatment greatest linear dimension (GLD), also choriocapillaries $0.99 \pm 0.84$ and outer retina $0.40 \pm 0.39$ of Post treatment area, while Post treatment retinal thickness on top of CNV279.0 \pm 56.90 .

Table (3): Comparison between pre and post according to treatment greatest linear dimension (GLD) (mm), treatment Area (mm2) and treatment retinal thickness on top of CNV (um).

\begin{tabular}{|l|c|c|c|c|c|c|}
\hline & $\begin{array}{c}\text { Pre } \\
(\mathbf{N = 2 0 )}\end{array}$ & $\begin{array}{c}\text { Post } \\
(\mathbf{N = 2 0})\end{array}$ & Mean Diff. & Diff.\% & t-test & p-value \\
\hline $\begin{array}{l}\text { Treatment greatest linear } \\
\text { dimension (GLD) (mm) }\end{array}$ & & & & & & \\
\hline Choriocapillaries & $4.36 \pm 1.84$ & $2.99 \pm 1.35$ & -1.37 & $-31.4 \%$ & 9.934 & $<0.001 * *$ \\
\hline Outer retina & $2.89 \pm 1.16$ & $1.74 \pm 1.18$ & -1.15 & $-39.8 \%$ & 6.718 & $<0.001 * *$ \\
\hline Treatment Area (mm2) & & & & & & \\
\hline Choriocapillaries & $1.41 \pm 1.13$ & $0.99 \pm 0.84$ & -0.42 & $-29.8 \%$ & 6.210 & $<0.001 * *$ \\
\hline Outer retina & $0.63 \pm 0.47$ & $0.40 \pm 0.39$ & -0.23 & $-36.5 \%$ & 8.452 & $<0.001 * *$ \\
\hline $\begin{array}{l}\text { Treatment retinal thickness on } \\
\text { top of CNV (um) }\end{array}$ & $488.3 \pm 176.99$ & $279.0 \pm 56.90$ & -209.3 & $-42.9 \%$ & 4.552 & $<0.001 * *$ \\
\hline
\end{tabular}

This table shows highly statistically significant difference between pre and post according to treatment greatest linear dimension (GLD) (mm), treatment Area (mm2) and treatment retinal thickness on top of CNV (um).

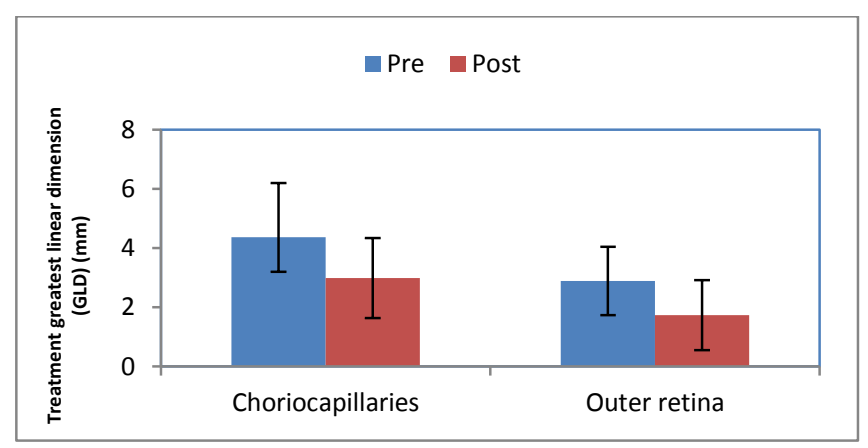

Figure (1): Bar chart between pre and post according to treatment greatest linear dimension (GLD) (mm).

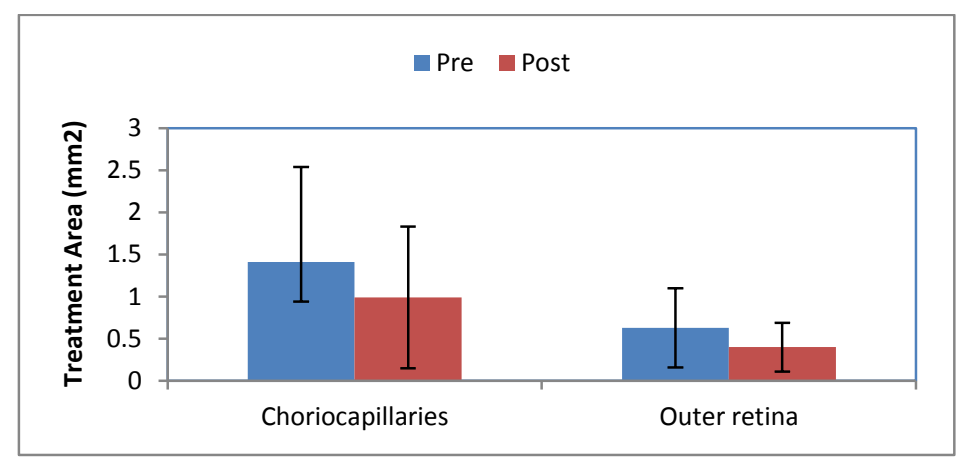

Figure (2): Bar chart between pre and post according to treatment Area (mm2). 


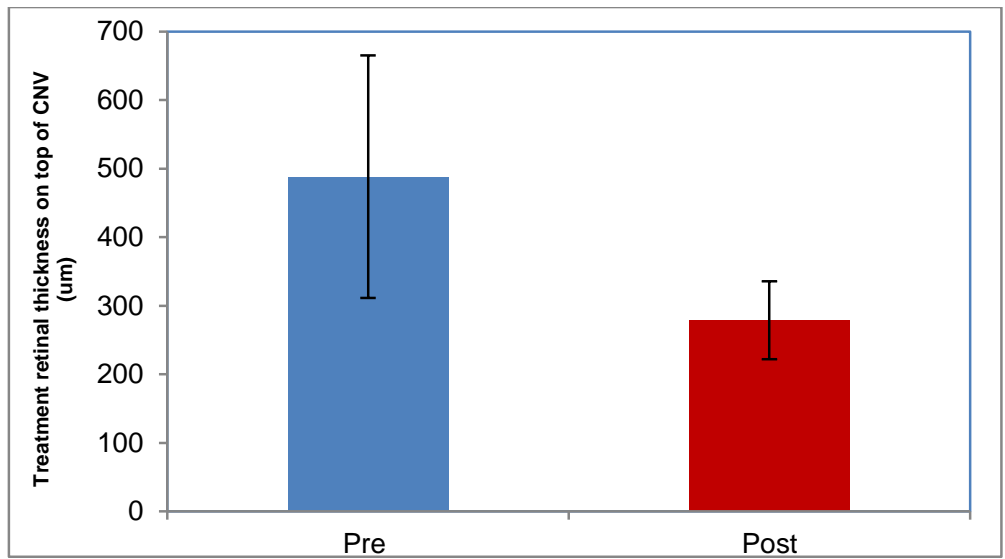

Figure (3): Bar chart between pre and post according to treatment retinal thickness on top of CNV (um).

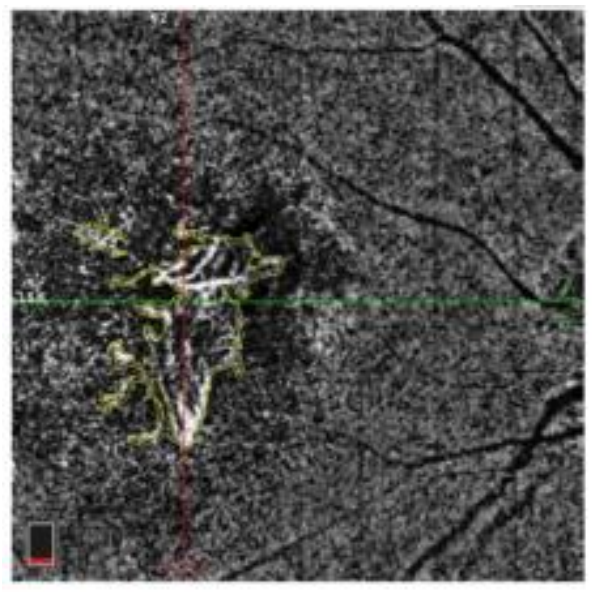

(A) Pre treatment

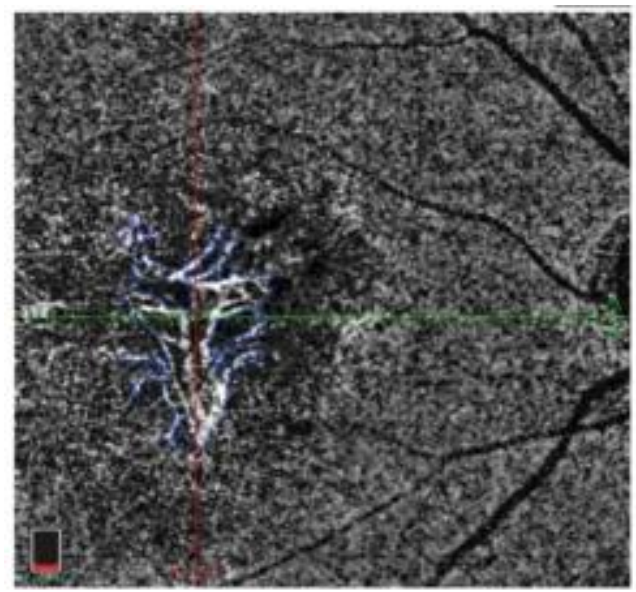

(B) Post treatment

Figure (4): Comparison between pre (A) (yellow line in the upward photo) and post treatment (B) (blue line in the downward photo) of the CNV area in the choriocapillaries (mm2) of the same patient.

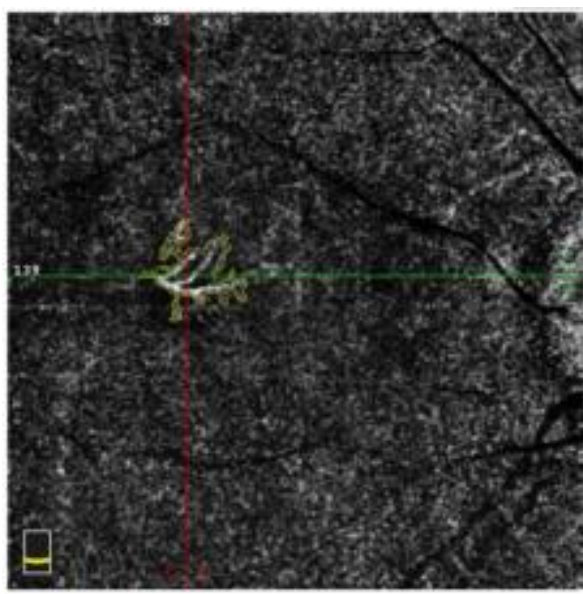

(A)pretreatment

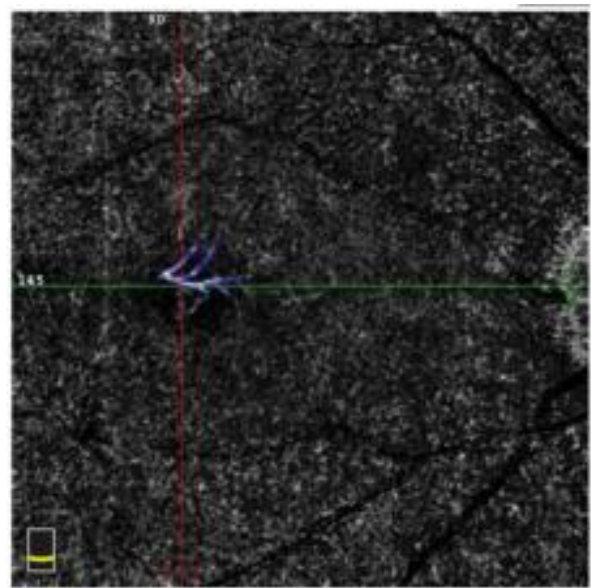

(B)post treatment

Figure (5): Comparison between pre (A) (yellow line in the upward photo) and post (blue line in the downward photo) treatment (B) of the CNV area in the outer retina ( $\mathrm{mm} 2)$ of the same patient. 
Effect of three Intravitreal Injections of Aflibercept on the CNV Guided by Optical Coherence...

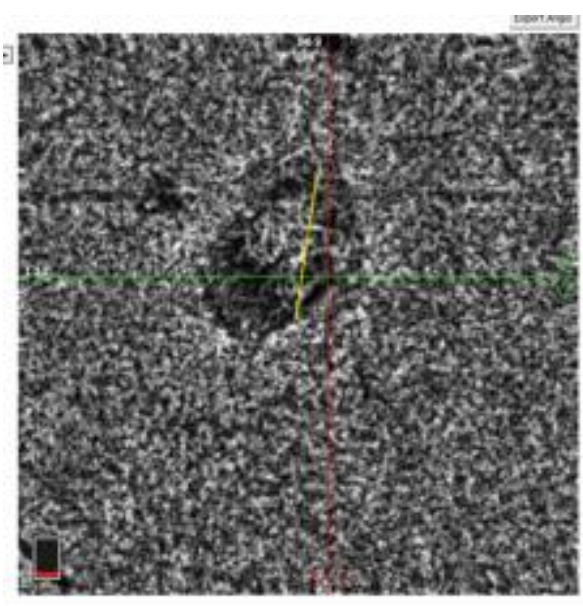

(A) pretreatment

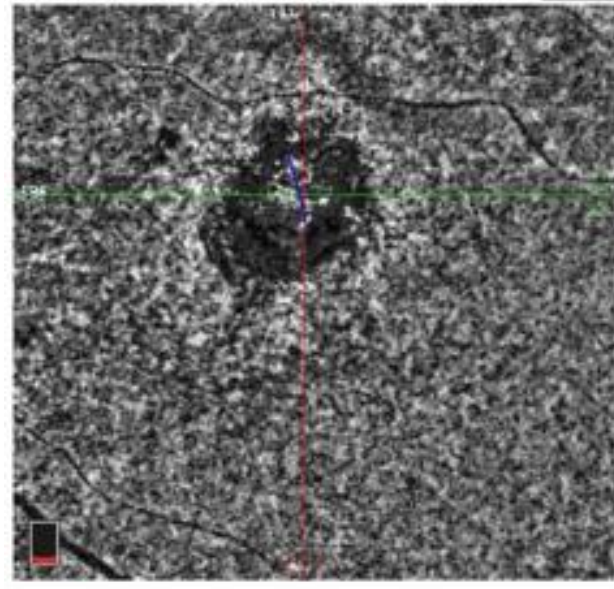

(B) posttreatment

Figure (6): Comparison between pre (A) (yellow line in the upward photo) and post (blue line in the downward photo) treatment (B) of greatest linear dimension (GLD) in the choriocapillaries (mm) of the same patient.

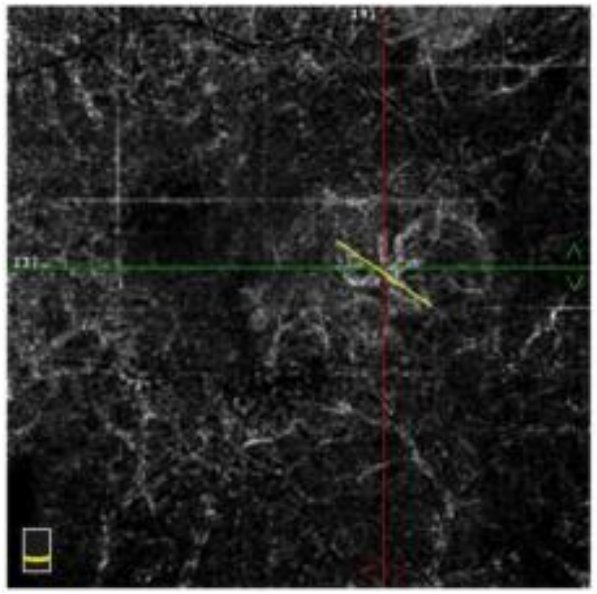

(A) pretreatment

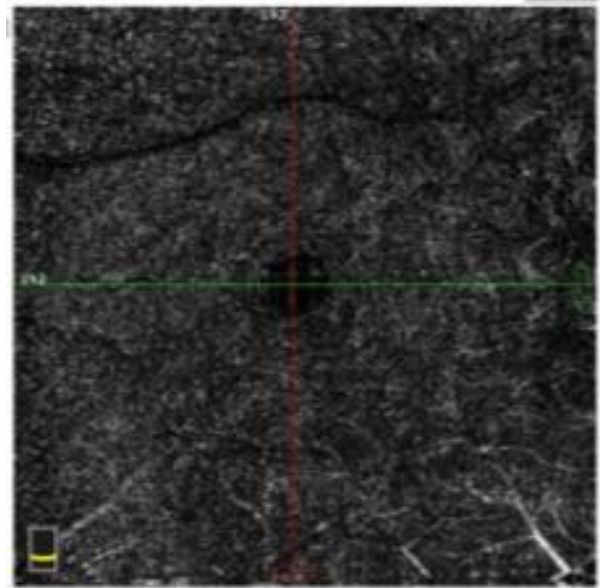

(B) posttreatment

Figure (7): Comparison between pre (A) (yellow line in the upward photo) and post treatment (B) of greatest linear dimension (GLD) in the outer retina $(\mathrm{mm})$ of the same patient, notice that: the downward photo shows a completely resolved CNV.

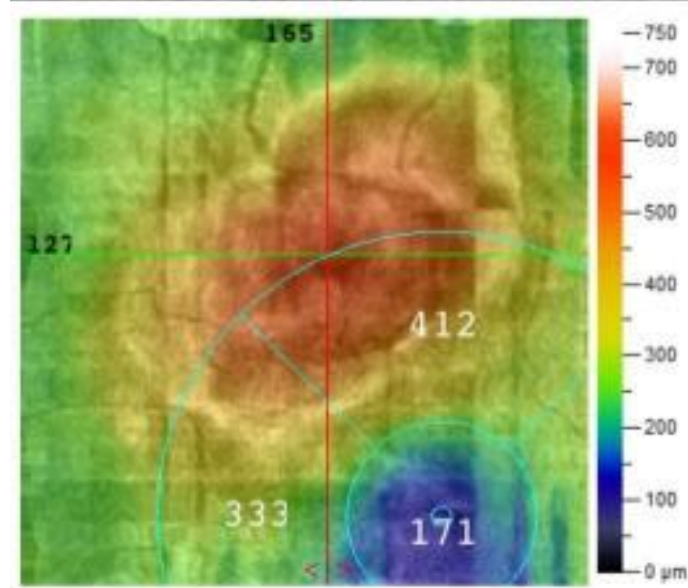

(A) pretreatmenr

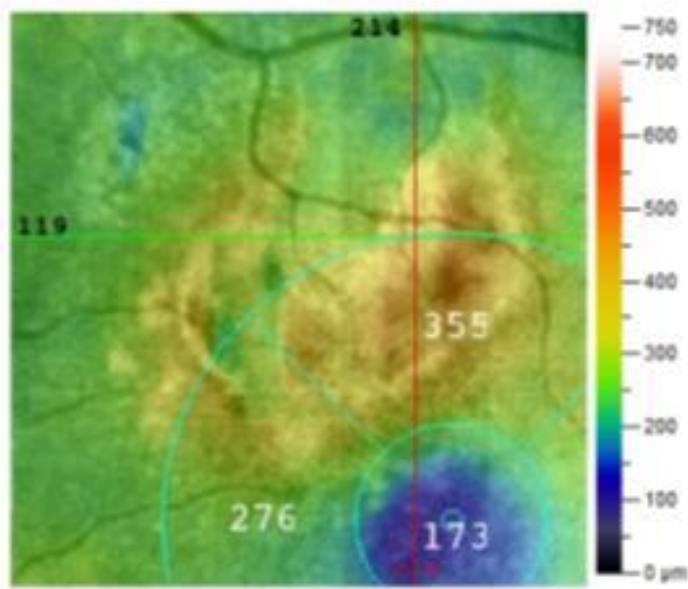

(B) posttreatment

Figure (8): Comparison between pre (A) (the upward photo) and post (the downward photo) treatment (B) retinal thickness on top of CNV (um) of the same patient. 


\section{Discussion:}

In the present study, OCTA scanning was done for eyes with wet AMD for anatomical assessment of $\mathrm{CNV}$ area size in both of (choriocapillaries and outer retina), GLD in both of (choriocapillaries and outer retina) and macular thickness on top of the $\mathrm{CNV}$, that will be done at baseline before starting intravitreal aflibercept treatment and at follow up one month after the loading dose (3 injections) of intravitreal aflibercept treatment.

In the present study, we found that the area size of the $\mathrm{CNV}$ had highly statistically significantly improved from mean value of [1.41 $\pm 1.13 \mathrm{~mm} 2]$ at baseline to $[0.99 \pm 0.84 \mathrm{~mm} 2]$ at follow up $(\boldsymbol{p}<0.001)$ and the mean value of change occurred was [-29.8\%] in choriocapillaries. Moreover, the area size of the CNV had highly statistically significantly improved from mean value of [0.63 $\pm 0.47 \mathrm{~mm} 2]$ at baseline to $[0.40 \pm 0.39 \mathrm{~mm} 2]$ at follow up $(\boldsymbol{p}<0.001)$ and the mean value of change occurred was $[-36.5 \%]$ in outer retina. The present study results typically agreed with those of Muakkassa et al. ${ }^{(9)}$ in which the mean value of change in the $\mathrm{CNV}$ area size in choriocapillaries had been improved to [-29.8\%] after intravitreal single dose of bevacizumab injection $(\boldsymbol{p}<0.001)$.

In the present study, we found that the GLD of the CNV had highly statistically significantly improved from mean value of $[4.36 \pm 1.84 \mathrm{~mm}]$ at baseline to $[2.99 \pm 1.35 \mathrm{~mm}]$ at follow up $(\boldsymbol{p}<0.001)$ and the mean value of change occurred was [-31.4\%] in choriocapillaries. Moreover, the GLD of the CNV had highly statistically significantly improved from mean value of $[2.89 \pm 1.16 \mathrm{~mm}]$ at baseline to $[1.74 \pm 1.18 \mathrm{~mm}]$ at follow up $(\boldsymbol{p}<0.001)$ and the mean value of change occurred was [-39.8\%] in outer retina. The present study results had been agreed with those of ${ }^{(9)}$ in which the mean value of change in the GLD of the CNV in choriocapillaries had been improved to [23.6\%] after intravitreal single dose of bevacizumab injection $(\mathrm{p}<0.001)$.

The difference in the results between both studies is suggested to be due to the following;

In Muakkassa et $\boldsymbol{a l} .{ }^{(9)}$ study the number of intravitreal injection was (a single dose) while in the present study (a 3 doses) had been injected, in addition to the difference of the anti-VEGF type had been used which were bevacizumab and aflibercept respectively. In the present study, we found that the retinal thickness on top of $\mathrm{CNV}$ had highly statistically significantly improved from mean value of $[488.3 \pm 176.99 \mu \mathrm{m}]$ at baseline to $[279.0 \pm 56.90 \mu \mathrm{m}]$ at follow up $(\boldsymbol{p}<0.001)$ and the mean value of change occurred was $[-209.3]=[-42.9 \%]$. This decrease in the retinal thickness on top of CNV agreed with Chun et al. ${ }^{(10)}$ results in which mean central foveal thickness (CFT) at baseline was [310.82 $\mu \mathrm{m}]$ and improved highly statistically significantly with mean value of change occurred was $[-119.44]=[-$ $38.42 \%$ ] $(\mathrm{p}<0.0001)$ at12 months follow up of bevacizumab intravitreal injection.

Also, this decrease in retinal thickness map agreed with Bolz et al. ${ }^{(11)}$ results in which mean CFT at baseline was $[324 \pm 94 \mu \mathrm{m}]$ and decreased significantly by $[-109 \pm 98 \mu \mathrm{m}]=[-33.64 \%]$ $(\mathrm{p}<0.0001)$ at month three of ranibizumab intravitreal injection.

The difference in the results between both studies is suggested to be due to the following;

Both Danny et al. ${ }^{(10)}$ and Bolz et al. ${ }^{(11)}$ studies had been measured the CFT which may not contain the juxtafoveal CNV, while in the present study we had measured the retinal thickness in the site top to the CNV where the maximum edema size usually present thus, the improvement in the thickness may increase. In addition to the difference of the anti-VEGF type had been used which were bevacizumab and ranibizumab respectively, while in the present study we had used aflibercept.

\section{Conclusion}

After 3 times intravitreal injections of aflibercept in cases of AMD, we concluded that, aflibercept is an effective treatment in AMD; it can decrease the area size, GLD and thickness of the CNV. OCTA is an effective tool that can provide a noninvasive method in the diagnosis and follow up of the activity and anatomical changes in AMD.

\section{References}

1. Mathenge W, Bastawrous A, Peto $\mathrm{T}$ et al. (2013): Prevalence of age related macular degeneration in Nakuru, Kenya: a cross sectional population based study. PLoS Med., 10: e1001393. 
Effect of three Intravitreal Injections of Aflibercept on the CNV Guided by Optical Coherence...

2. Wong TY, Chakravarthy U, Klein R et al. (2008): The natural history and prognosis of neovascular age related macular degeneration: a systematic review of the literature and metaanalysis. Ophthalmology, 115: 116-126.

3. Ferrara $N$ and Kerbel RS et al (2005): Angiogenesis as a therapeutic target. Nature, 438: 967-974.

4. Lanzetta P, Mitchell P, Wolf S et al. (2013): Different antivascular endothelial growth factor treatments and regimens and their outcomes in neovascular age-related macular degeneration: a literature review. $\mathrm{Br} \mathrm{J}$ Ophthalmol., 97: 1497-1507.

5. Ozkaya A, Alkin Z, Agca A et al. (2013): One-year results of treatment with bevacizumab alone or ranibizumab alone for low visual acuity due to neovascular agerelated macular degeneration. $\mathrm{J}$ Ocul Pharmacol Ther., 29: 865-869.

6. David J, Browning PK, Kaiser, PJ et al. (2012): Aflibercept for Age-Related Macular Degeneration: A Game-Changer or Quiet Addition. Am J Ophthalmol., 154 (2): 222226.

7. Chakravarthy U, Harding SP, Rogers CA et al. (2013): Alternative treatments to inhibit
VEGF in age related choroidal neovascularization: 2-year findings of the IVAN randomized controlled trial. IVAN study investigators. Lancet, 382: 1258-1267.

8. Talisa E, Romano A, Waheed NK et al. (2015): A review of optical coherence tomography angiography: International Journal of Retina and Vitreous, 119:101-110.

9. Muakkassa Nw, Chin At, Carlo $\mathrm{T}$ et al. (2015): Characterizing the effect of antivascular endothelial growth Factor therapy on treatment-naïve choroidal neovascularization using optical coherence tomography angiography. Retina, 35:22522259.

10. Chun Ng, Kwok AK, Tong JM et al. (2016): Bevacizumab for neovascular age-related macular degeneration in Chinese patients in clinical setting. Int J Ophthalmol., 9(3): 424430.

11. Bolz M, Simader C, Ritter M et al. (2010): Morphological and functional analysis of the loading regimen with intravitreal ranibizumab in neovascular age related macular degeneration. Br J Ophthalmol., 94(2):185189. 